\title{
EFFICIENT INFORMATION MAINTENANCE USING COMPUTATIONAL INTELLIGENCE IN THE MULTI-CLOUD ARCHITECTURE
}

\author{
Dr. Jennifer S. Raj, \\ Professor, Department of Electronics \& Communication Engineering, \\ Gnanamani College of Technology, \\ Namakal, India. \\ Email: jennifer.raj@gmail.com
}

\begin{abstract}
The multi cloud architecture improvises the constancy of the system associated with the cloud computing as they offer benefits such as the reducing the severity of the vendor lock in, which is the major impediment to the cloud service adoption. However the information maintenance in the multi cloud architecture is a very tedious and challenging as it inherits multitudes of complexity due to various services rendered, interfaces and technologies used. As storage is the fundamental usage of the cloud computing, the failures in the information maintenance (storage, securing, retrieval) in the traditional cloud computing has led to the development of different techniques that are highly reliable and flexible. The paper also scope in developing one such adaptable and highly secure information maintenance using the Cryptography integrated Computational intelligence (CICI) for application deployment in the multi cloud architecture. The proposed method is validated using the network simulator -Two, to verify the performance augmentation of the multi-cloud architecture in its information maintenance.
\end{abstract}

Keywords: Cloud System, Multi Cloud Architecture, Computational Intelligence, cryptography, Information Maintenance, Storage, Protection, Retrieval

\section{INTRODUCTION}

The cloud computing [1] is an upcoming technology that allows the resources, information and software to be shared. The cloud computing [2] is regarded as the well planned technology nowadays due to its service provisioning and the advanced storage methodologies. The cloud architecture is gaining very high importance recently and is becoming predominant on among various applications [3]. The benefits in the cloud [4]make more

ISSN: 2582-2640 (online) 
Journal of Soft Computing Paradigm (JSCP) (2019)

Vol.01/ No. 02

Pages: 113- 124

http://irojournals.com/jscp/

DOI: https://doi.org/10.36548/jscp.2019.2.006

and more users to adopt the cloud service which in result causes a huge amount of data flow in the cloud causing difficulties in managing the and maintaining the storage of the information's

The cloud paradigm [4] provides the multitudes of services such as the software, infrastructure, communication, network and the monitoring as service either under the public cloud paradigm that is shared among multiple of users or private cloud that is specifically used by one organization or group of similar organizations or the hybrid cloud that includes private cloud paradigm into the public cloud.

For the IT sectors in which the volume of the data are exponentially growing at a rapid pace, the hybrid and the multi cloud architecture have brought a new reality. Though the hybrid cloud paradigms are promising they do not work well or give assured success if failed to construct on the ground work supported by the robust data. So this makes the multi-cloud paradigm a well suited architecture for the aligning the data storage, build the applications properly and run them seamlessly. Primarily the multi cloud is preferred as it provides different cloud offerings, for the application associated with it and makes the applications run in multiple clouds. The multiple clouds are highly beneficial in terms of cost, speed and performance.

But the management/ maintenance of the data stored in the multi -cloud is the very tedious and challenging. So the paper utilizes the CICI-cryptography integrated Computational intelligence (genetic algorithm included with the elliptic curve Diffie Hellman) in multi cloud architecture to improve the maintenance of the information by providing a high reliable and more adaptable data storage, and retrieval.

The paper is arranged with the section 2 elaborating the related works done in favor of multi cloud architecture deployment, development and benefits, section 3 providing the frame work of the proposed method, section 4 . Explaining the results analysis for the proposed method and section 5. Provides the conclusion.

ISSN: 2582-2640 (online) 
Journal of Soft Computing Paradigm (JSCP) (2019)

Vol.01/ No. 02

Pages: 113- 124

http://irojournals.com/jscp/

DOI: https://doi.org/10.36548/jscp.2019.2.006

\section{RELATED WORKS}

The management of the information that is to be stored protected and retrieved requires an effective management system that side steps the complexities such as the technological variations, interfaces data loss and down time so several methods have been utilized to enhance the information maintenance in the cloud based system Naik et al [1]. In has paper details the "utilization of the swarm docker system that include several dependability characteristics to aid the development of the multi cloud dependable system.

Banyal, R. K., et al [2] has proffered a "highly reliable and a more secure cloud storage system to improve the data management in the cloud based systems" Ashalatha, et al [3] details the "algorithm for the data storage security in multi cloud architecture" Thakur et al details the "Framework to improve data integrity in multi cloud environment"

Horn et al [5] describes the "Cost Benefits of Multi-cloud Deployment of Dynamic Computational Intelligence Applications." Whereas Naik et al [6] in his paper details the "Docker container-based big data processing system in multiple clouds for everyone."

Ferry et al [7] in his paper presents the "model-driven provisioning, deployment, monitoring, and adaptation of multi-cloud systems." Pitchai, et al [8] provides the "methods to predict the availability and the integrity of the data in the cloud using the soft computing"

Mukhopadhyay et al [9] presents the "architecture that enables the Secure Cloud Computing with Elliptic Curve Cryptography" Junghanns et al [10] deals with the "methods in addressing the security and the privacy issues that are associated with the data storage in the public cloud services"

ISSN: 2582-2640 (online) 
Journal of Soft Computing Paradigm (JSCP) (2019)

Vol.01/ No. 02

Pages: 113- 124

http://irojournals.com/jscp/

DOI: https://doi.org/10.36548/jscp.2019.2.006
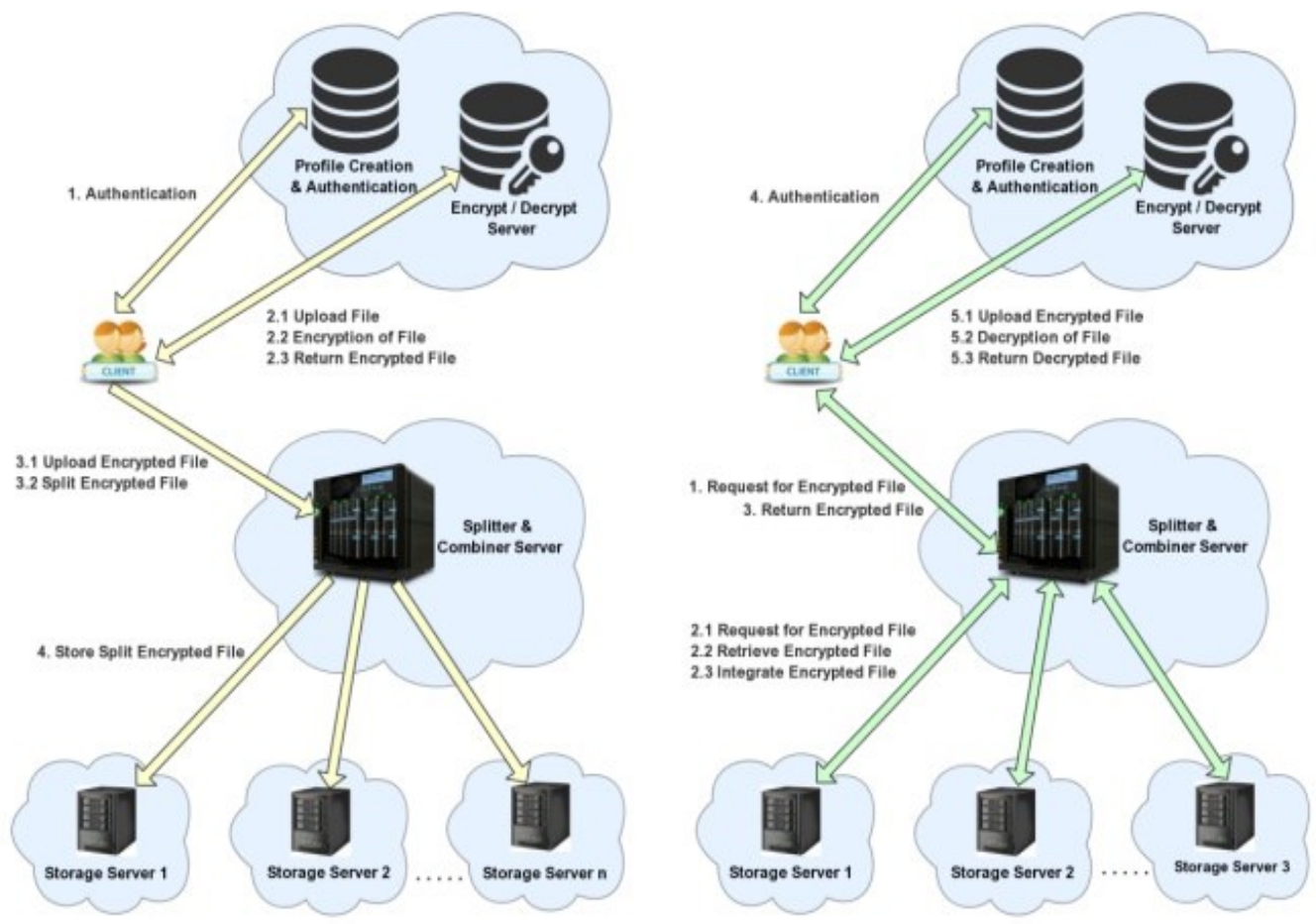

Fig.1. Information Storage and Retrieval with the Cryptography [10]

Casola, et al [11] et al the author proffers "MUSA deployer: deployment of multi-cloud applications." The Jiao et al [12] in his paper present the method of the "Multi-objective data placement for multi-cloud socially aware services." Raj et al [13], provides the "survey on the technologies, tools and methods for multiple cloud management" GR et al [14] provides the "the efficient security model in cloud computing based on soft computing techniques." , Iqbal et al [15] presents an "A provable and secure key exchange protocol based on the elliptical curve diffe-hellman for wsn." Raj, Jennifer S. et al [16] present the "comprehensive survey on the computational intelligence techniques as well as its applications." S. Smys. et al [17] present the utilization of the "cloud in the ERP to improve its sustainability"

ISSN: 2582-2640 (online)

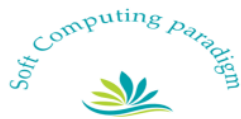


Journal of Soft Computing Paradigm (JSCP) (2019)

Vol.01/ No. 02

Pages: 113- 124

http://irojournals.com/jscp/

DOI: https://doi.org/10.36548/jscp.2019.2.006

\section{PROPOSED MODEL}

The cloud paradigm being a most successful and a promising methodology in the present days is utilized in variety of applications that fall under different domains. They are capable of handling variety of computing and storage necessities, paving way to build more complex systems that are supported by heterogeneous services provided by diverse service renders associated with the cloud offering the end users with the services that are value added. The multi cloud applications are the examples of one such application that involves the resources of the multiple cloud providers that are independent and does not require any co-operation among the involved service providers of the cloud [11]. The fig.2 below provides the block diagram of the proposed method.

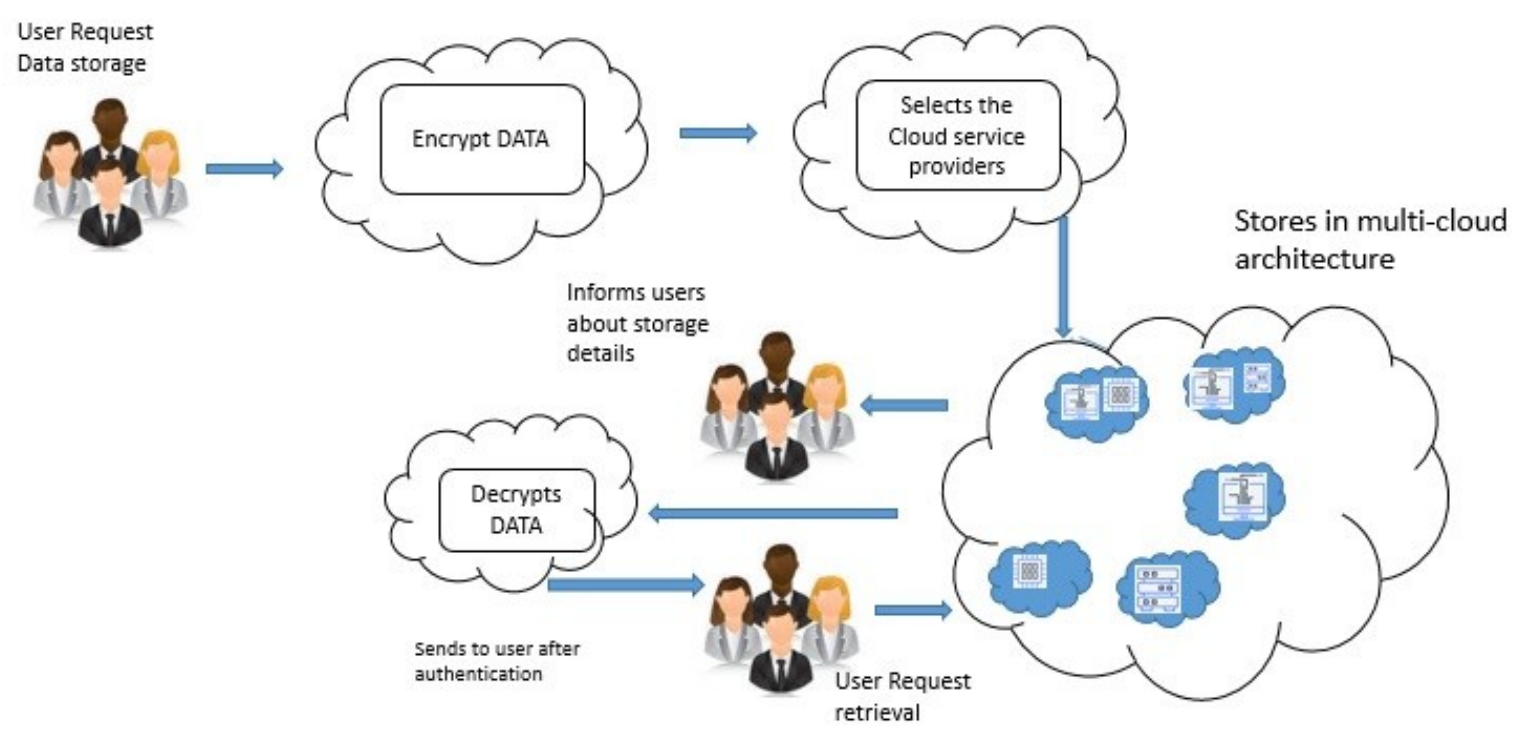

Fig.2 Proposed Block Diagram

ISSN: 2582-2640 (online) 
Journal of Soft Computing Paradigm (JSCP) (2019)

Vol.01/ No. 02

Pages: 113- 124

http://irojournals.com/jscp/

DOI: https://doi.org/10.36548/jscp.2019.2.006

The basic challenges in the deployment of the application in multi cloud architecture are

(i). Selection of the appropriate cloud services that satisfies the functional and the non-functional requirement of the application

(ii). Secured method of information storage and retrieval

(iii). Proper deployment planning enabling a migration of the application components across the cloud.

So the aim of the paper is have a proper deployment of applications in the multi cloud architecture and efficient maintenance of information storage in the multi cloud environment. So this achieved in the paper by employing the CICI- Elliptic-Diffie Hell Man integrated with the Genetic Algorithm. The paper employs the

1. Genetic algorithm [14] in the selection of the Service providers in cloud such that the functional and the nonfunctional requirements of the application are satisfied.

2. Elliptic-Diffie Hell Man [15] algorithm to secure the data storage and the retrieval.

3. The deployment planning of the multi cloud is handled by engaging the planner and he broker [11]

\subsection{GENETIC ALGORITHM FOR SERVICE PROVIDER SELECTION}

The genetic algorithm is a population based flexible method to identify the fittest individual. It is mainly used for solving the problems associated with the searching and the optimizations. It utilizes the basic of the survival of the fittest, natural selection and the natural population to find the more optimal solutions for the problem present in the real world. It involves the steps shown in the fig . 3 below to bring out the befitting solutions from the population.

ISSN: 2582-2640 (online)

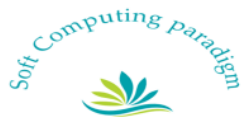


Journal of Soft Computing Paradigm (JSCP) (2019)

Vol.01/ No. 02

Pages: 113- 124

http://irojournals.com/jscp/

DOI: https://doi.org/10.36548/jscp.2019.2.006

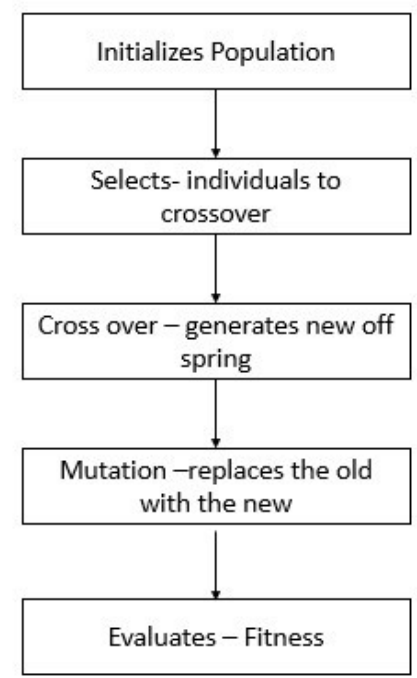

Fig .3 Step in Genetic Algorithm

Here the GA based search is employed to identify the most fitting resources from the multi cloud architecture for the user request on the storage. To identify the best resources the size of the data $\left(\right.$ data $\left._{\text {size }}\right)$ are enumerated maximum days of storage $\left(\max _{\text {days }}\right)$ is noted based on the information gathered from the users the genetic algorithm is applied to find out the more befitting resources $\left(B e_{s p}\right)$ for the information to be stored from the multi cloud architure by enumerating the cost of storage $\left(s_{\text {cost }}\right)$, the size of the storage $\left(S_{\text {size }}\right)$ and the availability $\left(S_{\text {avail }}\right)$. The Genetic algorithm applied identifies the optimal storage based on the user request.

\subsection{ELLIPTIC-DIFFIE HELL MAN FOR SECUIRNG THE INFORMATION}

The information to be stored cloud architecture are secured applying the Elliptic-Diffie Hellman algorithm to prevent the misuse of the confidential information's. The Elliptic-Diffie Hellman (EDH) is the basically a key agreement protocol that enables a two persons to safety and securely convey an information without any interference. For two persons to convey information. The EDH enables both the user and the service provider to

ISSN: 2582-2640 (online)

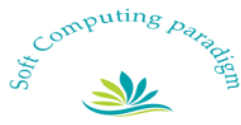


Journal of Soft Computing Paradigm (JSCP) (2019)

Vol.01/ No. 02

Pages: 113- 124

http://irojournals.com/jscp/

DOI: https://doi.org/10.36548/jscp.2019.2.006

generate their own public and the private keys and enables them to exchange the public keys on the channel that is insecure. Later the user calculates the shared key that is secret using his own private and the service provider's public key, the same is done by the service provider also. So this avoids the middle man to be unaware of the shared key that is secret.

1. Initially the user and the service provider enumerates their own public and the private key. Where the user public key is denoted as the $P_{u}=\operatorname{Pr}_{u} G$, where the $P r_{u}$ is the private key of the user and the $G$ is the base point on the same elliptic curve on the same finite field.

2. The service provider's public and the private key is denoted as $P_{S}=P r_{S} G$ and $P r_{S}$ the user and the service provider uses the same base point $G$.

3. The public key of the user and the service provider are exchanged over an insecure channel.

4. The user and the service provides enumerates the shared secret key $S s_{k e y U}=P r_{u} P_{S}$ and $S s_{k e y s}=P r_{S} P_{u}$

The shared secret key remains as an authentication between the user and the service provider, and protect the information from being misused or hacked.

\subsection{PROPOSED ALGORITHM FOR THE CICI FOR MULTI CLOUD ARCHITECTURE}

The process of information maintenance that includes the proper storage, protection and retrieval is accomplished using the Genetic algorithm that helps in identifying the prefect storage and utilizes the EDH algorithm to protect the data stored in the multi cloud architecture. The algorithm below in the fig .4 shows the step involved in the proposed method.

ISSN: 2582-2640 (online) 
Journal of Soft Computing Paradigm (JSCP) (2019)

Vol.01/ No. 02

Pages: 113- 124

http://irojournals.com/jscp/

DOI: https://doi.org/10.36548/jscp.2019.2.006

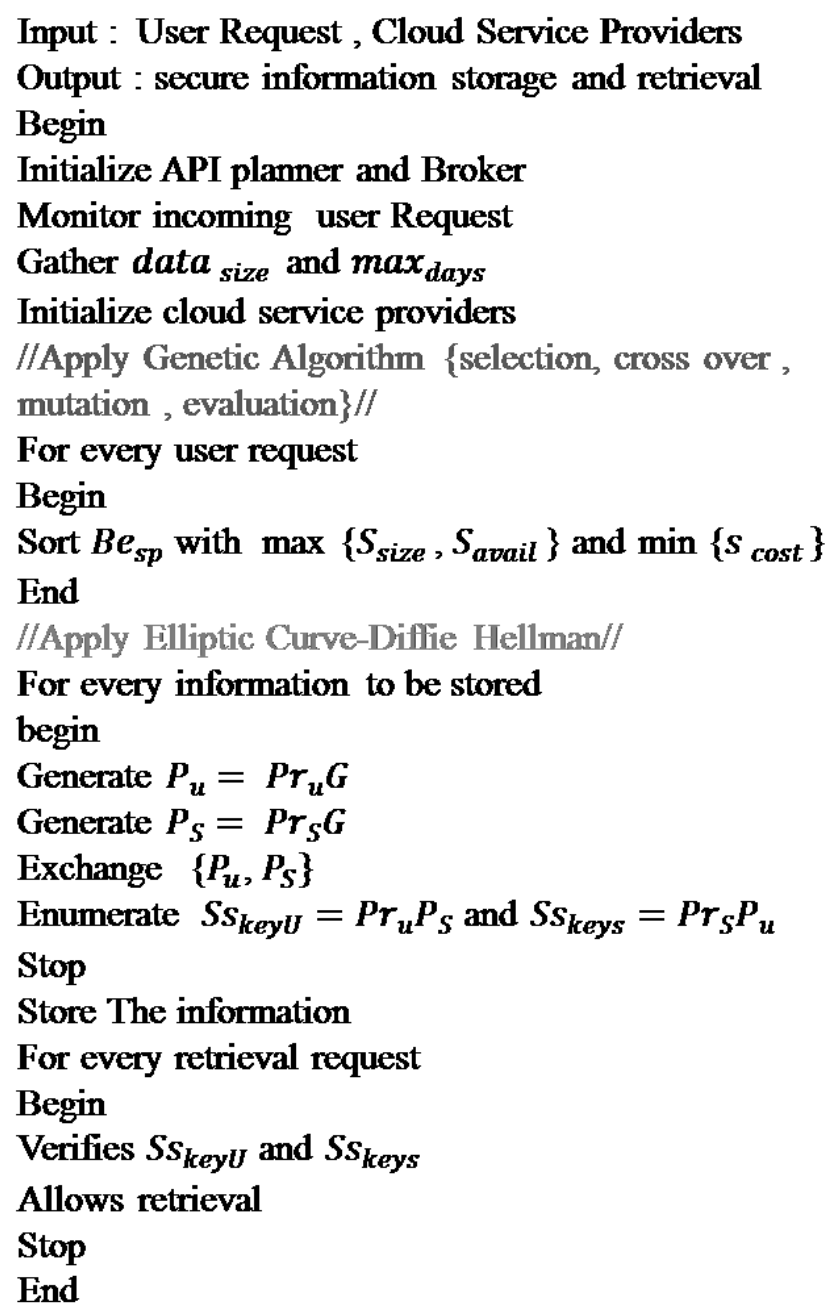

Fig.4.Proposed Algorithm

The deployment planning of the multi cloud architecture is done employing the planner and broker as proposed by the author Casola in [11]. The planner and the broker take the responsibility of preparing, implementing and managing the steadfastness of the plans involved in deployment. The planner API and the Broker API are employed in charge of the building deployment plan and monitoring respectively.

ISSN: 2582-2640 (online) 
Journal of Soft Computing Paradigm (JSCP) (2019)

Vol.01/ No. 02

Pages: 113- 124

http://irojournals.com/jscp/

DOI: https://doi.org/10.36548/jscp.2019.2.006

\section{RESULTS ANALYSIS}

The proposed method is validated using the network simulator-2 to enumerate the performance augmentation achieved in the multi cloud architecture in its information maintenance (protected storage and retrieval). The proposed method applies the GA and the EDH in identifying the proper storage for the user information in the cloud and enables a protected storage and retrieval respectively. The proposed method is evaluated along with the AVIP [8] for user request varying from 100 to 200 , of different sizes for the simulation time of 1000 s. The security provision and the cost of the information storage and the retrieval are evaluated for the above two method to bring out the performance upliftings achieved by the CICI (GA-EDH). The tabulation below in the table.1 shows the percentage of the security provisioning, the cost of the information maintenance and conveyance, the failures tolerated, expected additional waits etc.

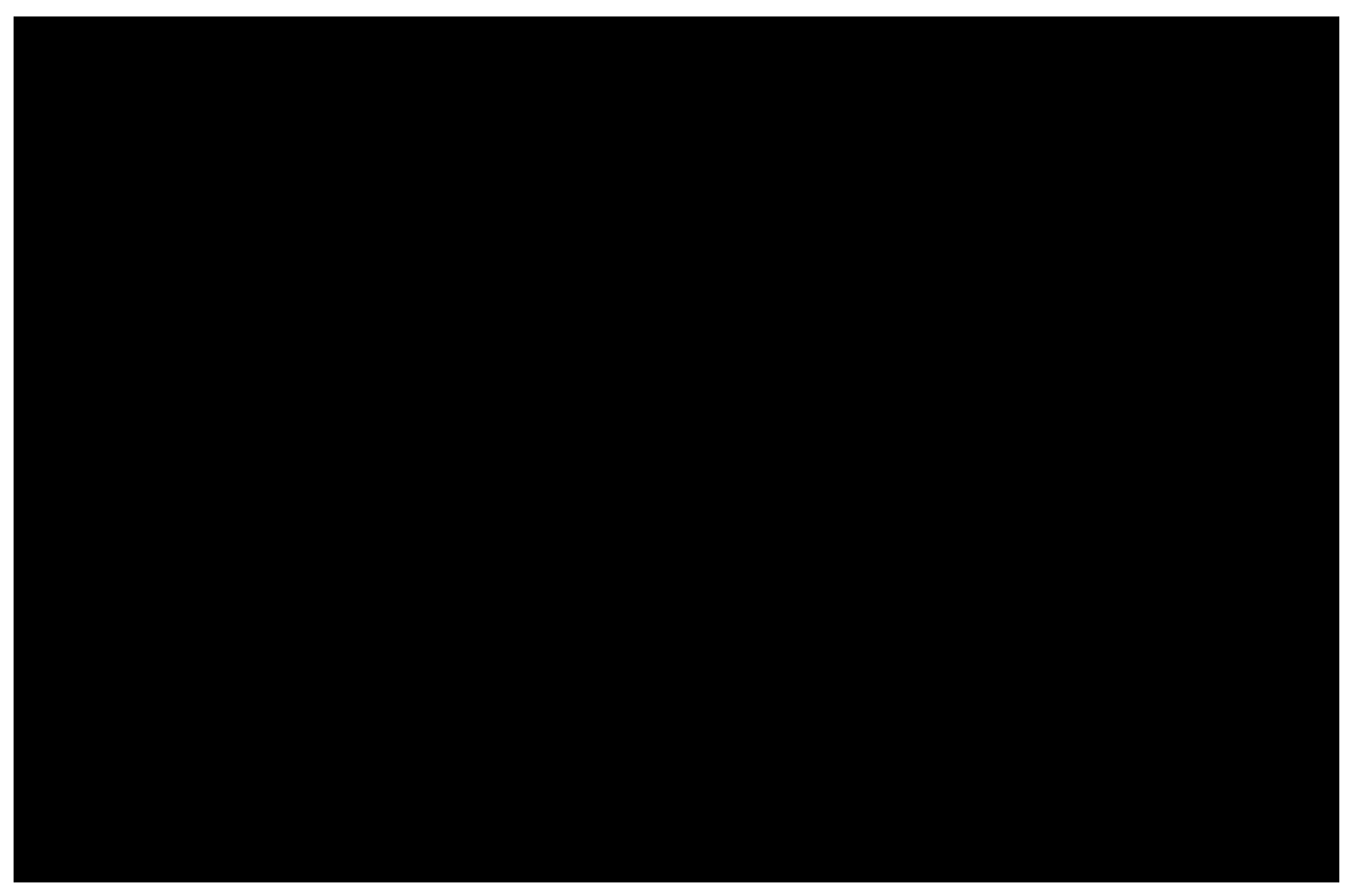

Table.1 The comparison of the Performance Augmentation

ISSN: 2582-2640 (online) 
Journal of Soft Computing Paradigm (JSCP) (2019)

Vol.01/ No. 02

Pages: 113- 124

http://irojournals.com/jscp/

DOI: https://doi.org/10.36548/jscp.2019.2.006

\section{CONCLUSION}

The information maintenance in the multi-cloud architecture is a tedious and challenging due to increasing number of adoption towards the cloud environment. So the paper has framed the cryptography included computational intelligence the GA with the EDH to support the proper information storage by selecting the befitting service provider and protecting the information storage and retrieval respectively. The CICI-GA WITH EDH is validated for the different number of user request of varying sizes using the network simulator-2 and compared with the AVIP. The results obtained shows that the proposed method provides augmented performance in the multi-cloud architecture for the information maintenance.

\section{References}

[1]. Naik, Nitin. "Applying computational intelligence for enhancing the dependability of multi-cloud systems using docker swarm." In 2016 IEEE Symposium Series on Computational Intelligence (SSCI), pp. 1-7. IEEE, 2016.

[2]. Banyal, R. K., V. K. Jain, and Pragya Jain. "Data Management System to Improve Security and Availability in Cloud Storage." In 2015 International Conference on Computational Intelligence and Networks, pp. 124-129. IEEE, 2015.

[3] Ashalatha, R', Jayashree Agarkhed, and Siddarama Patil. "Data storage security algorithms for multi cloud environment." In 2016 2nd International Conference on Advances in Electrical, Electronics, Information, Communication and Bio-Informatics (AEEICB), pp. 686-690. IEEE, 2016.

[4] Thakur, Anandita Singh, and P. K. Gupta. "Framework to improve data integrity in multi cloud environment." International Journal of Computer Applications 87, no. 10 (2014).

[5] Horn, Geir, Paweł Skrzypek, Katarzyna Materka, and Tomasz Przeździęk. "Cost Benefits of Multi-cloud Deployment of Dynamic Computational Intelligence Applications." In Workshops of the International Conference on Advanced Information Networking and Applications, pp. 1041-1054. Springer, Cham, 2019.

[6] Naik, Nitin. "Docker container-based big data processing system in multiple clouds for everyone." In 2017 IEEE International Systems Engineering Symposium (ISSE), pp. 1-7. IEEE, 2017.

ISSN: 2582-2640 (online) 
Journal of Soft Computing Paradigm (JSCP) (2019)

Vol.01/ No. 02

Pages: 113- 124

http://irojournals.com/jscp/

DOI: https://doi.org/10.36548/jscp.2019.2.006

[7] Ferry, Nicolas, Alessandro Rossini, Franck Chauvel, Brice Morin, and Arnor Solberg. "Towards modeldriven provisioning, deployment, monitoring, and adaptation of multi-cloud systems." In 2013 IEEE Sixth International Conference on cloud computing, pp. 887-894. IEEE, 2013.

[8] Pitchai, R., S. Babu, P. Supraja, and S. Anjanayya. "Prediction of availability and integrity of cloud data using soft computing technique." Soft Computing 23, no. 18 (2019): 8555-8562.

[9] Mukhopadhyay, Debajyoti, Akash Thakur, Nikhilesh Chaudhari, and Sani Nanekar. "Architecture to Implement Secure Cloud Computing with Elliptic Curve Cryptography." SmartCR 5, no. 3 (2015): 201208.

[10] Junghanns, Philipp, Benjamin Fabian, and Tatiana Ermakova. "Engineering of secure multi-cloud storage." Computers in Industry 83 (2016): 108-120.

[11] Casola, Valentina, Alessandra De Benedictis, Massimiliano Rak, Umberto Villano, Erkuden Rios, Angel Rego, and Giancarlo Capone. "MUSA deployer: deployment of multi-cloud applications." In 2017 IEEE 26th International Conference on Enabling Technologies: Infrastructure for Collaborative Enterprises (WETICE), pp. 107-112. IEEE, 2017.

[12] Jiao, Lei, Jun Lit, Wei Du, and Xiaoming Fu. "Multi-objective data placement for multi-cloud socially aware services." In IEEE INFOCOM 2014-IEEE Conference on Computer Communications, pp. 28-36. IEEE, 2014.

[13] Raj, Pethuru, and Anupama Raman. "Multi-cloud management: Technologies, tools, and techniques." In Software-Defined Cloud Centers, pp. 219-240. Springer, Cham, 2018.

[14] GR, Vijay, and A. Rama Mohan Reddy. "An efficient security model in cloud computing based on soft computing techniques." International Journal of Computer Applications 975 (2012): 8887.

[15] Iqbal, Ummer, and Saima Shafi. "A provable and secure key exchange protocol based on the elliptical curve diffe-hellman for wsn." In Advances in Big Data and Cloud Computing, pp. 363-372. Springer, Singapore, 2019.

[16] Raj, Jennifer S. "A COMPREHENSIVE SURVEY ON THE COMPUTATIONAL INTELLIGENCE TECHNIQUES AND ITS APPLICATIONS." Journal of ISMAC 1, no. 03 (2019): 147-159.

[17] Raj, Jennifer S., and S. Smys. "VIRTUAL STRUCTURE FOR SUSTAINABLE WIRELESS NETWORKS IN CLOUD SERVICES AND ENTERPRISE INFORMATION SYSTEM." Journal of ISMAC 1, no. 03 (2019): 188-204.

ISSN: 2582-2640 (online)

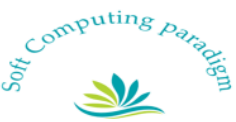

